\title{
REVIEW
}

\section{Chronic administration of anticonvulsants but not antidepressants impairs bone strength: clinical implications}

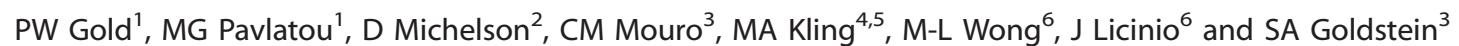

\begin{abstract}
Major depression and bipolar disorder are associated with decreased bone mineral density (BMD). Antidepressants such as imipramine (IMIP) and specific serotonin reuptake inhibitors (SSRIs) have been implicated in reduced BMD and/or fracture in older depressed patients. Moreover, anticonvulsants such as valproate (VAL) and carbamazepine (CBZ) are also known to increase fracture rates. Although $B M D$ is a predictor of susceptibility to fracture, bone strength is a more sensitive predictor. We measured mechanical and geometrical properties of bone in 68 male Sprague Dawley rats on IMIP, fluoxetine (FLX), VAL, CBZ, CBZ vehicle and saline $(S A L)$, given intraperitoneally daily for 8 weeks. Distinct regions were tested to failure by four-point bending, whereas load displacement was used to determine stiffness. The left femurs were scanned in a MicroCT system to calculate mid-diaphyseal moments of inertia. None of these parameters were affected by antidepressants. However, VAL resulted in a significant decrease in stiffness and a reduction in yield, and CBZ induced a decrease in stiffness. Only CBZ induced alterations in mechanical properties that were accompanied by significant geometrical changes. These data reveal that chronic antidepressant treatment does not reduce bone strength, in contrast to chronic anticonvulsant treatment. Thus, decreased BMD and increased fracture rates in older patients on antidepressants are more likely to represent factors intrinsic to depression that weaken bone rather than antidepressants per se. Patients with affective illness on anticonvulsants may be at particularly high risk for fracture, especially as they grow older, as bone strength falls progressively with age.
\end{abstract}

Translational Psychiatry (2015) 5, e576; doi:10.1038/tp.2015.38; published online 2 June 2015

\section{INTRODUCTION}

Recent data in large populations of older individuals indicate that specific serotonin reuptake inhibitors (SSRIs) are associated with the risk of decreased bone mineral density (BMD) and/or hip fracture. ${ }^{1-3}$ Other studies report that both SSRIs and tricyclic antidepressants (TCAs) increase the risk of hip fracture in older patients without commenting on $\mathrm{BMD}^{4-6}$ The impact of antidepressants on BMD is complicated by the fact that many papers also find that major depression is associated with loss of BMD per se. ${ }^{7-9}$ This loss of BMD in patients with major depression potentially reflects a number of hormonal alterations that may be risk factors for decreased BMD, including hypercortisolism, decreased secretion of growth hormone and hypothalamic hypogonadism. ${ }^{10-12}$ In addition, melancholic patients with major depression have around-the-clock increases in plasma and CSF norepinephrine secretion, ${ }^{13}$ and recent data indicate that sympathetic drive contributes to bone loss. ${ }^{14}$ Depressed patients are also in a mild proinflammatory state, characterized by changes such as increased plasma IL- $6^{15}$ and acute phase protein levels. ${ }^{16}$ It is well known that inflammation has a major role in some forms of osteoporotic bone loss, ${ }^{17}$ especially at the hip. In our premenopausal women with major depression, bone loss was considerably greater at the hip than at the spine, ${ }^{7}$ in contrast to the usual pattern of greater loss of vertebral bone in common forms of osteoporosis. This pattern is more compatible with inflammatory causes of osteoporosis. Thus, inflammation may be particularly important in the bone loss of depressive illness.

Anticonvulsants are also well known to contribute to reductions in BMD and increase the rate of bone fracture. These compounds are used not only to treat epilepsy, but also major depressive illness as well. Several factors may contribute to the increased fracture risk reported in patients with epilepsy. Epileptic seizures may per se lead to injury. In addition, subjects with epilepsy, especially if children, may have insufficient dietary intake of vitamin D, limited sun exposure, and decreased ambulation and physical activity, all nonspecific factors that can lead to impaired bone structure and function. ${ }^{18}$ However, anticonvulsants may also affect bone mass by specific mechanisms. Their mechanisms include impaired calcium absorption, induction of microsomal enzymes with consequent accelerated hepatic catabolism of vitamin $D_{1}$ secondary hypoparathyroidism, and osteomalacia. $^{19-21}$

There is extensive and long-term clinical use of antidepressant and antiepileptic agents, and drug-induced osteoporosis is a preventable condition. We thus decided to test whether chronic administration of antidepressants and anticonvulsants to rats may affect mechanical strength, an extremely important determinant of fracture risk in low impact trauma. We know several mechanisms by which anticonvulsants can contribute to low BMD or bone fracture, but knowing more about their impact on

\footnotetext{
${ }^{1}$ Clinical Neuroendocrinology Branch, National Institute of Mental Health, National Institutes of Health, Bethesda, MD, USA; ${ }^{2}$ Merck, Whitehouse Station, NJ, USA; ${ }^{3}$ Orthopaedic Research Laboratories, University of Michigan, Ann Arbor, MI, USA; ${ }^{4}$ Behavioral Health Service, Philadelphia VA Medical Center, Philadelphia, PA, USA; ${ }^{5}$ Department of Psychiatry, University of Pennsylvania School of Medicine, Philadelphia, PA, USA and ${ }^{6}$ Department of Translational Medicine, John Curtin School of Medical Research, Australian National University, Canberra, ACT, Australia. Correspondence: Dr PW Gold, Clinical Neuroendocrinology Branch, National Institute of Mental Health, National Institutes of Health, 10 Center Drive, Room 2D46, Bethesda, MD 20892, USA.

E-mail: philipgold@mail.nih.gov
}

Received 30 June 2014; accepted 19 December 2014 


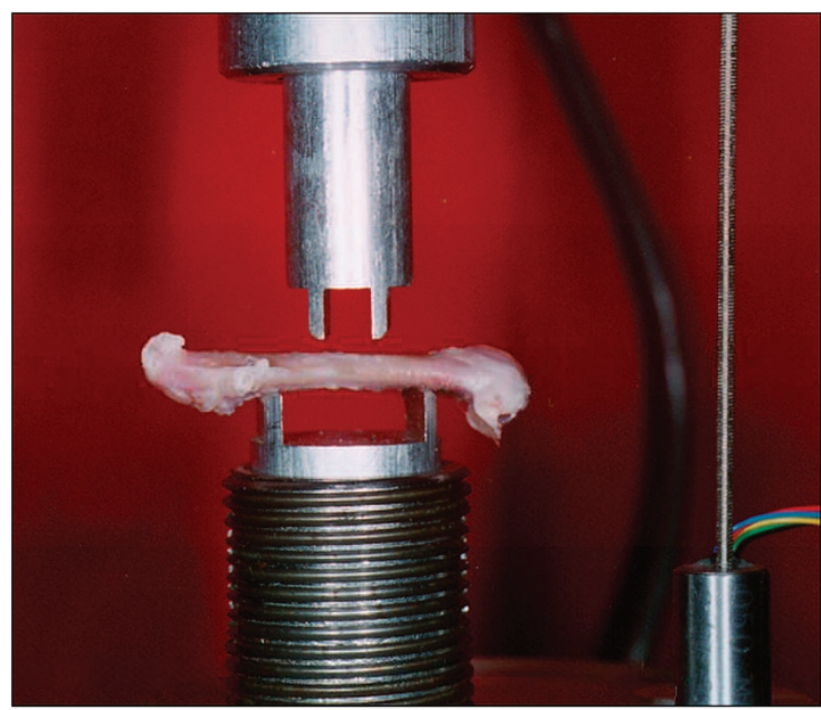

Figure 1. The femurs were loaded to failure by a custom designed four-point bending fixture secured within the structure of an MTS materials testing system. The region of the mid-diaphysis subjected to load between the four loading points corresponded precisely to the volume of bone evaluated by MicroCT.

specific parameters of bone strength may help contribute to methods of reducing the risk to bone health posed by anticonvulsants.

None of the factors promoting bone loss attributable to anticonvulsants have yet been found as a consequence of antidepressant treatment. Showing whether or not antidepressants decrease bone strength could help clarify the extent to which low BMD or fracture in depression can be, in part, attributable to medication, or is an intrinsic component of depressive illness. Bone strength may be more important than BMD in conferring susceptibility to bone fracture, so that BMD and bone strength are not necessarily synonymous. Therefore, it is of substantial clinical importance to establish whether and how chronic administration of antidepressants to the rat directly affects bone strength.

\section{MATERIALS AND METHODS}

Drug treatment

Sixty-eight male Sprague Dawley rats were treated either with the antidepressants imipramine (IMIP) or fluoxetine (FLX); with the anticonvulsants valproate (VAL) or carbamazepine (CBZ); or given a sham treatment via daily intraperitoneal injections for 8 weeks. A saline (SAL) sham treatment was used as the control for the IMIP, FLX and VAL groups, while carbamazepine vehicle (CBZ-V) was used as the control for the CBZ group. The FLX group was administered $1 \mathrm{mg} \mathrm{kg}^{-1}$ body weight of fluoxetine; the IMIP group was given $5 \mathrm{mg} \mathrm{kg}^{-1}$ of IMIP; the VAL group received 250 $\mathrm{mg} \mathrm{kg}^{-1}$ of valproate, all in $0.5 \mathrm{ml}$ saline vehicle. The SAL group was given $0.5 \mathrm{ml}$ saline alone. The CBZ group was treated with $40 \mathrm{mg} \mathrm{kg}^{-1}$ body weight of CBZ in a 1-ml propylene glycol/ alcohol/saline vehicle. The CBZ-V group received a sham treatment of $1 \mathrm{ml}$ of CBZ-V alone.

\section{Mechanical testing}

The right femurs from 53 rats, which had been stored fresh-frozen, were tested to failure in four-point bending using an MTS servohydraulic testing machine (Minneapolis, MN, USA) at a constant displacement of rate of $5 \mathrm{~mm} \mathrm{~s}^{-1}$ (see Figure 1). Loaddisplacement data were acquired and used to determine loads

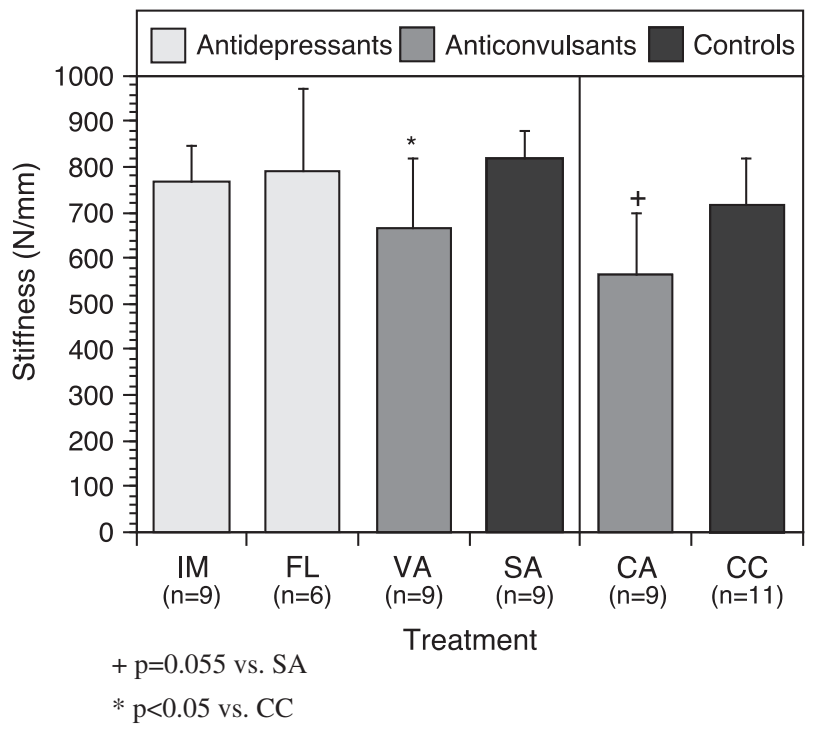

Figure 2. Anticonvulsant drug therapy demonstrated a significant decrease in whole bone stiffness. ${ }^{+} P=0.055$ vs $S A .{ }^{*} P<0.05$ vs CC. CA, carbamazepine; CC, controls; FL, fluoxetine; IM, imipramine; SA, saline; VA, valproate.

and displacements to both yield and failure. Stiffness was calculated as the slope of the linear portion of the loaddisplacement curve.

\section{Geometrical analysis}

The left femurs from 68 rats were scanned in a MicroCT system, and three-dimensional digital images were reconstructed at a resolution of $50 \mu \mathrm{m}$. The images were thresholded to distinguish bone from non-bone voxels, and the analysis region was defined as the mid-50\% region of the bone (mid-diaphysis). Crosssectional area, cortical thickness and moments of inertia were determined for each slice, and then averaged along the length of the analysis region. Moment of inertia is a mathematical representation of the distribution of bone material away from the neutral bending axis of the bone, and is directly related to mechanical properties in bending (Please see Appendix 1 for a further explication of this term).

\section{Statistical analysis}

A one-way analysis of variance was used to compare the FLX, IMIP and VAL groups with the SAL group, and to compare the CBZ and CBZ-V groups. Tukey's post hoc test was used for comparisons between groups with $P<0.05$ considered statistically significant.

\section{RESULTS}

Three months of daily treatment with IMIP and FLX had no impact on stiffness, bone yield or geometric properties of bone consisting of moment of inertia, cortical thickness and cross-sectional area (Figure 2).

In general, anticonvulsant drug therapy causes a decrease in mechanical properties of whole bones. Both the VAL and CBZ groups exhibited significant decreases in stiffness and yield load (see Figures 2 and 3). The VAL group exhibited an 18.5\% reduction in stiffness and a $25.3 \%$ reduction in yield load when compared with the SAL group. Treatment with CBZ resulted in a $21.3 \%$ decrease in stiffness and $26.5 \%$ decrease in yield load when compared with the CBZ-V group. Similar trends were seen in failure load results, although the comparison was only statistically significant between the CBZ and CBZ-V groups. 


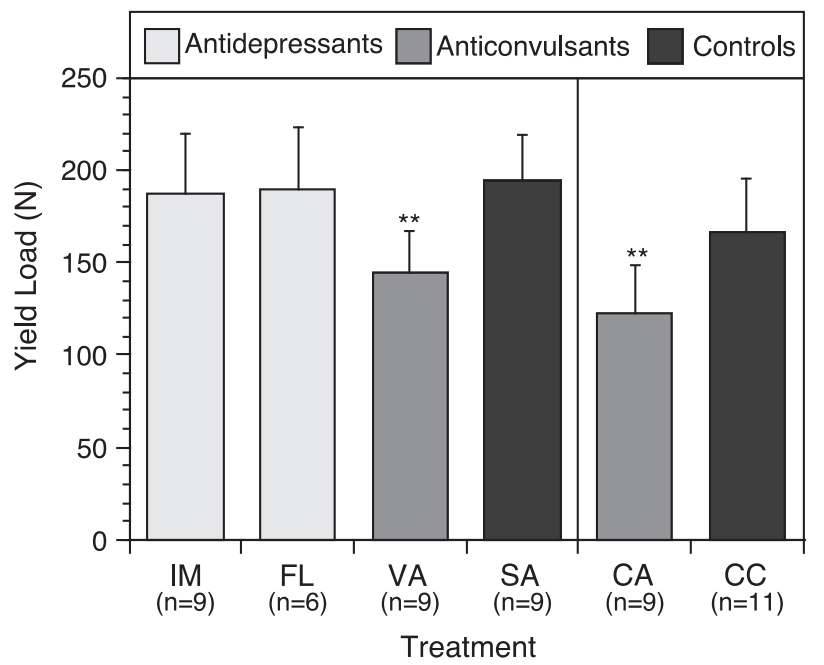

$* * \mathrm{p}<0.005$ vs. control

Figure 3. Similar to the results for stiffness, the anticonvulsants significantly reduced the load to yield in femurs. ${ }^{* *} P<0.005$ vs control. CA, carbamazepine; CC, controls; FL, fluoxetine; IM, imipramine; SA, saline; VA, valproate.

These alterations in mechanical properties were accompanied by significant geometrical changes only in the CBZ group (see Table 1). Cross-sectional area, moment of inertia and cortical thickness were significantly decreased in the CBZ group when compared with the CBZ-V group. The CBZ group exhibited an $18.3 \%$ reduction in moment of inertia when compared with the CBZ-V group, whereas the VAL group only showed a $7.7 \%$ decrease when compared with the SAL group (see Figure 4).

\section{DISCUSSION}

Eight-week-old male Sprague Dawley rats were treated either with the antidepressants, IMIP or FLX; anticonvulsants, VAL or CBZ; or given a sham treatment, saline SAL or CBZ vehicle via daily intraperitoneal injections for 8 weeks. The right femur was tested to failure in four-point bending. Load-displacement data were used to determine stiffness, and loads and displacements to both yield and failure. The left femurs were scanned in a MicroCT system to calculate cross-sectional area, cortical thickness and moments of inertia of the mid-diaphyseal region. Neither IMIP nor FLX had any significant effects on cross-sectional area, moment of inertia and cortical thickness. On the other hand, both anticonvulsants adversely influenced multiple determinants of bone strength.

Table 1. Geometrical properties of antidepressant- and anticonvulsant-treated rats

\begin{tabular}{lllc}
\hline & $C S A\left(\mathrm{~mm}^{2}\right)$ & $C T(\mathrm{~mm})$ & Ixx $\left(\mathrm{mm}^{4}\right)$ \\
\hline IM $(n=15)$ & $6.27 \pm 0.49$ & $0.696 \pm 0.052$ & $9.54 \pm 1.35$ \\
FL $(n=12)$ & $6.48 \pm 0.50$ & $0.725 \pm 0.052$ & $9.75 \pm 1.67$ \\
VA $(n=9)$ & $6.36 \pm 0.36$ & $0.740 \pm 0.095$ & $9.32 \pm 0.94$ \\
SA $(n=12)$ & $6.55 \pm 0.34$ & $0.715 \pm 0.026$ & $10.10 \pm 0.34$ \\
CA $(n=9)$ & $5.53 \pm 0.46^{* *}$ & $0.640 \pm 0.044^{*}$ & $7.78 \pm 1.03^{* *}$ \\
CC $(n=11)$ & $6.23 \pm 0.33$ & $0.671 \pm 0.020$ & $9.52 \pm 1.24$ \\
\hline
\end{tabular}

Abbreviations: $C A$, carbamazepine; CC, controls; CSA, cross-sectional area $\mathrm{CT}$, cortical thickness; $\mathrm{FL}$, fluoxetine; IM, imipramine; Ixx, moment of inertia along $x-x$ axis; $S A$, saline; VA, valproate. All values expressed as mean \pm s.d. ${ }^{*} P<0.05$ vs $C C .{ }^{* *} P<0.005$ vs $C$.

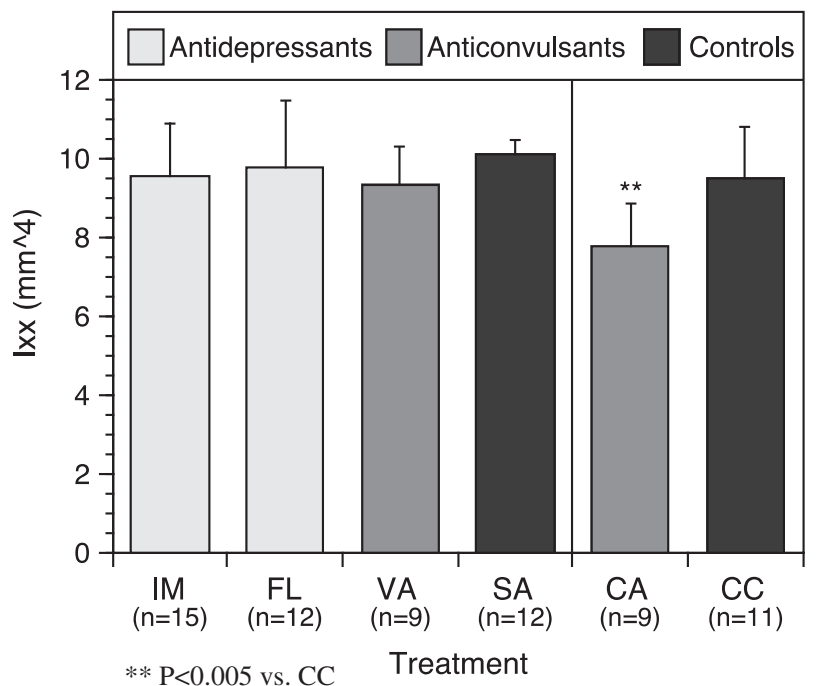

Figure 4. Only the anticonvulsant $\mathrm{CBZ}$ demonstrated a significant reduction in the moment of inertia, reflecting a change in the crosssectional geometry. ${ }^{* *} P<0.005$ vs CC. CA, carbamazepine; CC, controls; $\mathrm{FL}$, fluoxetine; IM, imipramine; $\mathrm{SA}$, saline; VA, valproate.

\section{SSRIs and TCAs: putative effects on BMD and hip fracture}

Diem et al. ${ }^{1}$ studied 2722 older women prospectively over a 5 -year period and concluded that SSRIs led to a significant reduction in BMD at the hip. This finding could be complicated by the fact that patients with depressive illness may lose bone faster than matched controls. Moreover, its functional significance is not clear as loss of BMD does not always correlate precisely with bone strength of relevance to the liability to fracture (vide infra).

Haney et al. ${ }^{2}$ studied 5995 men 65 years and older crosssectionally, showing that SSRIs were also associated with significant losses of BMD at the hip and spine, while subjects on TCAs or trazadone had no loss of BMD. Richards et al. ${ }^{3}$ studied 5008 community dwelling adults age 50 and above prospectively over a period of five years and also showed a clinically-relevant association between SSRI use, BMD loss at the hip, and a trend for BMD loss at the spine. SSRI treatment was also associated with twofold increased incidence of fragility fracture greater at the hip than at the spine.

Three studies have suggested that both SSRls and TCAs are associated with increased risk of hip fracture. Liu et al. ${ }^{5}$ studied a group of 8239 patients aged 66 years and older and found a twofold increase in hip fracture for both drugs. Similarly, Ziere et al. ${ }^{6}$ studied 1289 elderly subjects and found a greater than twofold increase in hip fracture for both drugs. None of these studies examined measures of BMD.

Bone has functional serotonergic system and responds to norepinephrine as well

In vitro and in vivo data in experimental animals indicate a functioning serotonin system in bone. Osteoblast and osteoclasts both express a serotonin transporter system, with mechanisms for responding to and taking up serotonin. ${ }^{22}$ Functional receptors for serotonin and the serotonin transporter have been identified in osteoblasts, osteoclasts and osteocytes. Serotonin has been shown to induce murine osteocytes. Serotonin and human osteoclast differentiation in vitro. The SSRI FLX inhibits osteoblast differentiation and osteoclast differentiation, ${ }^{23}$ effects that would have opposing effects on BMD.

Serotonin transporter (5HTT) knockout is associated with decreased bone accrual during growth. The authors suggest that this might relate to an effect in the $5 \mathrm{HTT}$ in reducing skeletal responsiveness to mechanical loading. However, using the 
Table 2. General effects

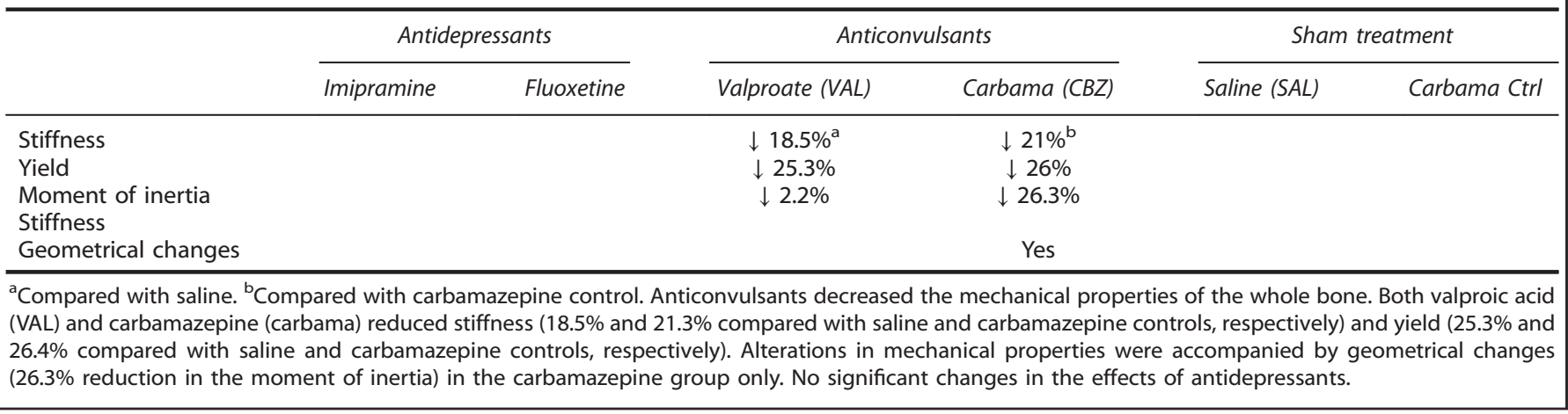

established loading model, $5 \mathrm{HTT}-/-$ and $5 \mathrm{HTT}+/+$ mice were exposed to identical osteogenic stimuli, no influence of the null mutation was found on skeletal growth.

Activation of the sympathetic nervous system promotes bone resorption. ${ }^{14,24}$ Yirmiya et al..$^{25}$ found that sympathetic nervous system activation in response to behavioral stressors causes bone loss in association with behavioral features of depression. A tricyclic mediated increase in bone fracture is not necessarily compatible with the clinical findings that TCAs are associated with an increase in the fracture rat via actions on the noradrenergic system. Thus, TCAs reduce sympathetic outflow significantly on one hand, while serving as norepinephrine reuptake blockers on the other. Moreover, even if tricyclics did increase availability of NE at receptors located on bone, their effects are so numerous that this effect alone might not necessarily lead to pathologic bone loss.

Despite responsiveness to monoaminergic inputs, loss of BMD and fractures may not be related to antidepressants

We have previously noted that even young, premenopausal women with major depression have significant losses in BMD greater at the hip than at the spine. Many other studies find reduced $B M D$ in both male and female populations of patients with depressive illness (for example, 7-9). The etiology of this loss of BMD is not known, but, as noted earlier, is likely to represent many factors, including a proinflammatory state, which is associated with preferential loss of bone at the hip compared with vertebral loss. The activation of the HPA axis and sympathetic nervous system, as well as inhibition of the growth hormone and gonadal axes could also contribute.

The data presented here suggest that antidepressants do not contribute to bone fractures after minimal trauma in older patients as these drugs do not reduce bone strength. Thus, increased fracture in older patients on antidepressants is likely to reflect factors intrinsic to depression itself. Accordingly, more older depressed patients will be on antidepressants than non-depressed patients, and hence, the fracture rate will be greater in patients on antidepressant treatment. Thus, preclinical studies showing that serotonin systems exist within bone and that bone is responsive to noradrenergic input do not, in themselves, implicate antidepressants in the diathesis to bone fracture in older depressed patients.

Clinical implications: relationship between bone density, bone strength and susceptibility to fracture-who should be watched carefully

The assumption that BMD equates with bone strength and resistance to fracture has not been fully validated, especially in younger individuals. ${ }^{26}$ Rather, resistance to fracture is a composite of its microarchitecture, accumulated microscopic damage and the quality of collagen, mineral crystal size and bone turnover. Many lines of evidence challenge the generally accepted orthodoxy that bone density is the best way to assess strength of bone. Indeed, denser bone is not always stronger. This discrepancy came to light with the use of sodium fluoride to treat osteoporosis. Although sodium fluoride produced large increases in density, fluoride made the bone more brittle because it changed the quality of the mineral and rendered it more susceptible to fracturing, especially in vertebral fracture regions. Thus BMD cannot always be considered a straightforward surrogate marker for bone strength and resistance to fracture, ${ }^{26}$ though the two are likely to be related.

Young bone and older bone differ in strength, even with similar bone density. Hence, young bone is stronger than older bone across all levels of bone mass. Moreover, clinical studies showed that the drugs approved for treating osteoporotic fractures generally fare better than we would expect from their effects on bone density. ${ }^{27}$ Thus, bones become stronger before they grow more dense. These data suggest that premenopausal women with loss of BMD are not necessarily more susceptible to fracture because of other factors associated with younger age such as high quality collagen. However, they are losing BMD faster than nondepressed controls and should be followed closely after menopause, when vulnerability to fracture increases because of loss of strength and of estrogen.

Like BMD, bone strength decreases progressively as a function of age. In older individuals, decreased bone strength increases fracture risk. As BMD loss is greater in premenopausal women at the hip than at the spine, fractures may be a particular problem for older women who suffer from depression, a premise validated by clinical studies. Almost all of the papers cited earlier about antidepressant use and fractures in older women specifically indicated a preferential effect on hip fractures over fractures of the spine. $^{4,5}$

\section{Anticonvulsants}

Anticonvulsant drugs such as CBZ and VAL are well known to affect BMD by altering the biochemical and mineral composition of bone tissue, and have been associated with a higher predisposition to bone fracture. ${ }^{28}$ These have been hypothesized to be caused by a vitamin D deficiency similar to osteomalacia. ${ }^{19} \mathrm{~A}$ recent study suggested that decreased BMD in patients treated with VAL might also be associated with increased bone resorption. VAL and CBZ may also interact with glucocorticoids in promoting bone loss. $^{29}$

In contrast to antidepressants, we report here that anticonvulsant treatments have a substantial effect on mechanical and geometrical properties of bone (Table 2). The detrimental effects of CBZ on mechanical properties of bone are paralleled by a similar alteration in moment of inertia, indicating that this drug affects both the geometry of bone and its material properties. In 
contrast, decreases in mechanical properties caused by VAL were not associated with concomitant changes in cross-sectional geometry. Therefore, this drug treatment is likely to exert its effect on bone properties through changes in material properties of the bone tissue and not geometrical alterations. Previous clinical studies on the effects of VAL and CBZ on BMD in humans reported that VAL monotherapy, but not CBZ, significantly reduces axial and appendicular BMD. ${ }^{18}$ This indicates that material property alterations in VAL-treated animals may be related, in part, to changes in mineralization. These results portray a potentially important association between anticonvulsant therapy and bone mass, and may reveal a novel mechanism affecting its regulation.

Anticonvulsants such as VAL and CBZ are often used not only in patients with epilepsy, but also in patients with bipolar disorder. Despite the fact that BMD and bone strength do not always correlate, BMD should be followed in depressed patients on anticonvulsants, as bone strength is likely to correlate with BMD, at least partially. The combination of depressive illness, anticonvulsants and old age may be particularly toxic to bone health.

\section{CONFLICT OF INTEREST}

The authors declare no conflict of interest.

\section{ACKNOWLEDGMENTS}

We acknowledge the substantial contributions of Erica Smith and Cameron Mouro for conducting many of the MicroCT and mechanical tests. We also acknowledge Mark Stock and Rick Zanecki for their technical assistance. This work was supported by funding from the Clinical Neuroendocrinology Branch, National Institute of Mental Health, NIH AR34399, the Whittaker Foundation and a Multidisciplinary Research Training Grant in Aging from the National Institute of Aging, NIA T32 AG00114 and a GAANN Fellowship.

\section{REFERENCES}

1 Diem SJ, Blackwell TL, Stone KL, Yaffe K, Haney EM, Bliziotes MM et al. Use of antidepressants and rates of hip bone loss in older women: the study of osteoporotic fractures. Arch Intern Med 2007; 167: 1240-1245.

2 Haney EM, Chan BK, Diem SJ, Ensrud KE, Cauley JA, Barrett-Connor E et al. Association of low bone mineral density with selective serotonin reuptake inhibitor use by older men. Arch Intern Med 2007; 167: 1246-1251.

3 Richards JB, Papaioannou A, Adachi JD, Joseph L, Whitson HE, Prior JC et al. Effect of selective serotonin reuptake inhibitors on the risk of fracture. Arch Intern Med 2007; 167: 188-194.

4 Hubbard R, Farrington P, Smith C, Smeeth L, Tattersfield A. Exposure to tricyclic and selective serotonin reuptake inhibitor antidepressants and the risk of hip fracture. Am J Epidemiol 2003; 158: 77-84.

5 Liu B, Anderson G, Mittmann N, To T, Axcell T, Shear N. Use of selective serotoninreuptake inhibitors or tricyclic antidepressants and risk of hip fractures in elderly people. Lancet 1998; 351: 1303-1307.

6 Ziere G, Dieleman JP, van der Cammen TJ, Hofman A, Pols HA, Stricker BH. Selective serotonin reuptake inhibiting antidepressants are associated with an increased risk of nonvertebral fractures. J Clin Psychopharmacol 2008; 28: 411-417.

7 Michelson D, Stratakis C, Hill L, Reynolds J, Galliven E, Chrousos G et al. Bone mineral density in women with depression. N Engl J Med 1996; 335: 1176-1181.

8 Schweiger U, Weber B, Deuschle M, Heuser I. Lumbar bone mineral density in patients with major depression: evidence of increased bone loss at follow-up. Am J Psychiatry 2000; 157: 118-120.

9 Eskandari F, Martinez PE, Torvik S, Phillips TM, Sternberg EM, Mistry S et al. Low bone mass in premenopausal women with depression. Arch Intern Med 2007; 167: 2329-2336.

10 Gold PW, Goodwin FK, Chrousos GP. Clinical and biochemical manifestations of depression. Relation to the neurobiology of stress (1). N Engl J Med 1988; 319: 348-353.
11 Gold PW, Goodwin FK, Chrousos GP. Clinical and biochemical manifestations of depression. Relation to the neurobiology of stress (2). N Engl J Med 1988; 319: 413-420.

12 Kling MA, Roy A, Doran AR, Calabrese JR, Rubinow DR, Whitfield HJ Jr. et al. Cerebrospinal fluid immunoreactive corticotropin-releasing hormone and adrenocorticotropin secretion in Cushing's disease and major depression: potential clinical implications. J Clin Endocrinol Metab 1991; 72: 260-271.

13 Gold PW, Wong ML, Goldstein DS, Gold HK, Ronsaville DS, Esler M et al. Cardiac implications of increased arterial entry and reversible 24-h central and peripheral norepinephrine levels in melancholia. Proc Natl Acad Sci USA. 2005; 102: 8303-8308.

14 Elefteriou F, Ahn JD, Takeda S, Starbuck M, Yang X, Liu X et al. Leptin regulation of bone resorption by the sympathetic nervous system and CART. Nature 2005; 434: 514-520.

15 Alesci S, Martinez PE, Kelkar S, llias I, Ronsaville DS, Listwak SJ et al. Major depression is associated with significant diurnal elevations in plasma interleukin-6 levels, a shift of its circadian rhythm, and loss of physiological complexity in its secretion: clinical implications. J Clin Endocrinol Metab 2005; 90: 2522-2530.

16 Kling MA, Alesci S, Csako G, Costello R, Luckenbaugh DA, Bonne O et al. Sustained low-grade pro-inflammatory state in unmedicated, remitted women with major depressive disorder as evidenced by elevated serum levels of the acute phase proteins C-reactive protein and serum amyloid A. Biol Psychiatry 2007; 62: 309-313.

17 Tamura T, Udagawa N, Takahashi N, Miyaura C, Tanaka S, Yamada Y et al. Soluble interleukin-6 receptor triggers osteoclast formation by interleukin 6. Proc Natl Acad Sci USA. 1993; 90: 11924-11928.

18 Sheth RD, Wesolowski CA, Jacob JC, Penney S, Hobbs GR, Riggs JE et al. Effect of carbamazepine and valproate on bone mineral density. J Pediatr 1995; 127: 256-262.

19 Hustmyer FG, Peacock M, Hui S, Johnston CC, Christian J. Bone mineral density in relation to polymorphism at the vitamin D receptor gene locus. J Clin Invest 1994; 94: $2130-2134$.

20 Jones G, Sambrook PN. Drug-induced disorders of bone metabolism. Incidence, management and avoidance. Drug safety 1994; 10: 480-489.

21 Sato Y, Kondo I, Ishida S, Motooka H, Takayama K, Tomita Y et al. Decreased bone mass and increased bone turnover with valproate therapy in adults with epilepsy. Neurology 2001; 57: 445-449.

22 Bliziotes MM, Eshleman AJ, Zhang XW, Wiren KM. Neurotransmitter action in osteoblasts: expression of a functional system for serotonin receptor activation and reuptake. Bone 2001; 29: 477-486.

23 Battaglino R, Fu J, Spate U, Ersoy U, Joe M, Sedaghat L et al. Serotonin regulates osteoclast differentiation through its transporter. J Bone Miner Res 2004; 19: 1420-1431.

24 Takeda S, Elefteriou F, Levasseur R, Liu X, Zhao L, Parker KL et al. Leptin regulates bone formation via the sympathetic nervous system. Cell 2002; 111: 305-317.

25 Yirmiya R, Goshen I, Bajayo A, Kreisel T, Feldman S, Tam J et al. Depression induces bone loss through stimulation of the sympathetic nervous system. Proc Natl Acad Sci USA 2006; 103: 16876-16881.

26 Stenstrom M, Olander B, Lehto-Axtelius D, Madsen JE, Nordsletten L, Carlsson GA Bone mineral density and bone structure parameters as predictors of bone strength: an analysis using computerized microtomography and gastrectomyinduced osteopenia in the rat. J Biomech 2000; 33: 289-297.

27 Licata A. Bone density vs bone quality: what's a clinician to do? Cleve Clin J Med 2009; 76: 331-336.

28 Akin R, Okutan V, Sarici U, Altunbas A, Gokcay E. Evaluation of bone mineral density in children receiving antiepileptic drugs. Pediatr Neurol 1998; 19: 129-131.

29 Ohnishi T, Ichikawa Y. Direct inhibitions of the activities of steroidogenic cytochrome P-450 mono-oxygenase systems by anticonvulsants. J Steroid Biochem Mol Biol 1997; 60: 77-85.

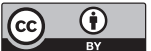

This work is licensed under a Creative Commons Attribution 4.0 International License. The images or other third party material in this article are included in the article's Creative Commons license, unless indicated otherwise in the credit line; if the material is not included under the Creative Commons license, users will need to obtain permission from the license holder to reproduce the material. To view a copy of this license, visit http://creativecommons.org/licenses/ by/4.0/ 


\section{APPENDIX}

The moment of inertia of an object about a given axis describes how difficult it is to change its angular motion about that axis. Therefore, it encompasses not just how much mass the object has overall, but how far each bit of mass is from the axis. The further out the object's mass is, the more rotational inertia the object has, and the more torque (force $\times$ distance from axis of rotation) is required to change its rotation rate. For example, consider two hoops, $A$ and $B$, made of the same material and of equal mass. Hoop $A$ is larger in diameter but thinner than $B$. It requires more effort to accelerate hoop A (change its angular velocity) because its mass is distributed farther from its axis of rotation: mass that is farther out from that axis must, for a given angular velocity, move more quickly than mass closer in. So in this case, hoop A has a larger moment of inertia than hoop B. 